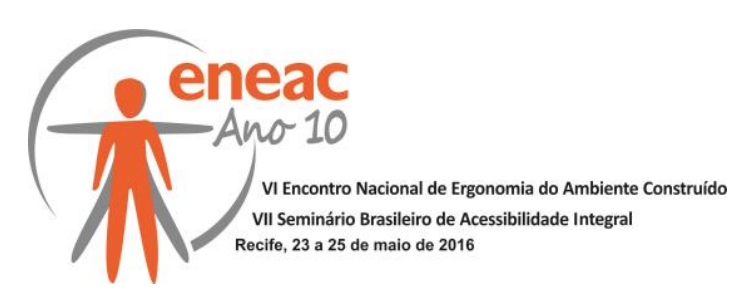

\title{
EXPERIÊNCIAS DA UFC NO CAMINHO DA ACESSIBILIDADE INTEGRAL
}

\author{
SANTIAGO, Zilsa Maria Pinto (1);
}

(1) Universidade Federal do Ceará, Doutora

e-mail: zilsa@arquitetura.ufc.br

\begin{abstract}
RESUMO
O presente texto tem por objetivo apresentar as experiências pontuais e institucionais ocorridas na Universidade Federal do Ceará (UFC), nos últimos quinze anos, pertinentes à temática da Acessibilidade Integral, sob vários pontos de vista, numa tentativa de trabalhar em vários eixos de ação, envolvendo diversos cursos e, consequentemente, buscar se aproximar de soluções mais amplas que envolvem a acessibilidade e a inclusão. Apresenta vários percursos na construção de uma cultura de inclusão, mostrando as dificuldades e avanços, exemplificando algumas atividades coletivas e outras pontuais, que vão agregando contribuições para somar nessa construção desejada.
\end{abstract}

Palavras chave: Acessibilidade integral; arquitetura; inclusão e transdisciplinaridade.

\begin{abstract}
This text aims to present individual and institutional experiences in the Federal University of Ceará (UFC) in the past fifteen years, relevant to the subject of Full Accessibility, in several respects, in an attempt to work on several lines of action, involving various courses and, therefore, seek to get closer to broader solutions that involve accessibility and inclusion. Offers various routes in building a inclusion culture, showing the difficulties and advances, exemplifying some collective activities and other specific, adding contributions to add to this desired culture.
\end{abstract}

Keywords: integral accessibility; architecture; inclusion and transdisciplinarity.

\section{RESUMO EXPANDIDO}

O relato trata das questões de acessibilidade e inclusão na Universidade Federal do Ceará, onde se iniciaram de forma pontual, em diferentes cursos, por iniciativas particulares de professores e alunos em seus ambientes de trabalho, sala de aula, pesquisa e extensão. No Departamento de Arquitetura e Urbanismo (DAU), especificamente, a temática começou a ser desenvolvida pontualmente, no Trabalho de Final de Curso (1994) da aluna Virgínia Hatsue, sobre uma "residência adaptada". Neste estudo a aluna passou um mês utilizando cadeira de rodas em vários locais da cidade para aproximar-se das dificuldades do cotidiano de um usuário de cadeira de rodas. Em 2000, foi criado o Projeto de Extensão "Acessibilidade em escolas de ensino fundamental e médio das redes públicas estadual e municipal", sob minha coordenação. Importante ressaltar que a ideia do projeto surgiu fora dos muros da Universidade, por solicitação do movimento V.I.D.A. (Vida, Independência, Dignidade e Ação), de forma a se trabalhar os espaços de inclusão. Em reunião do movimento, chegou-se a decisão de começar pela escola primária, considerando-se ser este o primeiro lugar de socialização e formação de cidadania. O Projeto contou inicialmente com a colaboração das arquitetas Célia Perdigão Coutinho e Virgínia Hatsue Sawaky, como 


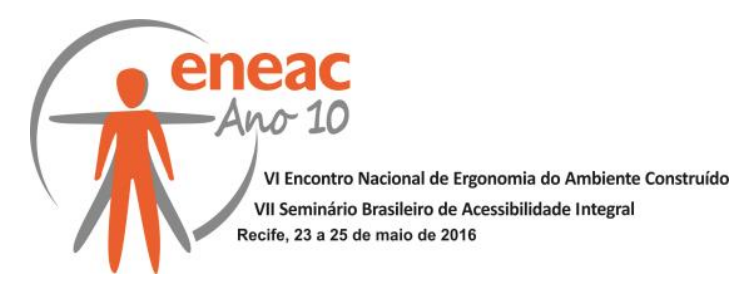

voluntárias; da professora Nadja Pinho Pessoa, também voluntária e representante do V.I.D.A.; de alunos bolsistas e alunos voluntários do curso de Arquitetura e Urbanismo e da aluna de Psicologia, também voluntária, Gerlene Lima. As participações de Nadja e Gerlene, como usuárias de cadeira de rodas, foi de fundamental importância para crescimento e sensibilização do grupo no sentido de compreender melhor as reais dificuldades que passam as pessoas com deficiência no espaço construído não acessível.

Este projeto, que continua até hoje, tem como objetivo principal desempenhar o papel da UFC, atendendo à comunidade cearense, por meio de ações no campo da Arquitetura voltadas para o desenho universal, bem como a partir do estudo sistematizado da acessibilidade ao edifício, ser uma visão crítica sobre a importância do assunto, dentro e fora do curso de Arquitetura e Urbanismo. A atuação do projeto visa ainda fazer o reconhecimento das condições de acessibilidade das escolas de ensino fundamental e médio, envolvendo alunos no trato das questões de uma realidade social. No mesmo período inicial do projeto de extensão, foi desenvolvido na disciplina de Arquitetura de Interiores o projeto da "Casa Segura", por solicitação dos médicos ortopedistas e traumatologistas para a mostra da Sociedade Brasileira de Ortopedia e Traumatologia Regional Ceará - SBOT-Ce (2000). Ressalta-se que a solicitação era também um alerta para os profissionais que lidam com a concepção de espaços, pois os médicos afirmavam que seus pacientes são acidentados, muitas vezes, em função de barreiras no espaço edificado. O projeto de extensão sobre acessibilidade nas escolas públicas foi objeto da minha dissertação de mestrado defendida na FAUUSP em 2005, tendo no seu escopo a análise de vinte escolas municipais construídas no período de 1990 a 2003, que se inicia pós homologação da primeira versão da NBR 9050/1985 até a data da pesquisa. Esse estudo teve como fundamentação os trabalhos de Dreyfuss (1955); Goldsmith (1976); Aino (1978); Croney (1978); Diffrient (1981); Pheasant (1986); Conde (1994); Steinfeld (1994); Ubierna (1997); Bahia et al (1998); Cohen (1999); Boueri (1999); Preiser (1988; 2001); Sanoff (2001); Ornstein e Roméro (2003); Dischinger e Bins Ely (2004); Cambiaghi (2004); Lopes (2004). Neste estudo foi sistematizado o procedimento de análise e seus resultados contribuem para outras pesquisas na academia como também recomendações para políticas públicas. Voltando a relato do projeto de extensão, este passou por várias fases, dentre elas, a de realização de convênio com a Prefeitura Municipal de Fortaleza em 2007/2008, envolvendo dez bolsistas, alunos do curso de Arquitetura e Urbanismo, desenvolvendo projeto de adaptação em 39 escolas municipais. Os projetos foram entregues à Secretaria Municipal de Educação de Fortaleza, tendo como propósito de subsidiar os processos de adaptação e serem incorporados em reformas das escolas.

Atualmente, a abordagem do projeto consiste em duas vertentes: a primeira, que dá continuidade aos trabalhos de vistoria técnica das condições de acessibilidade espacial das escolas, que este ano, está sendo desenvolvido em parceria com o Ministério Público Estadual no Projeto "Ministério Público pela Educação Básica de qualidade" (2016) com recursos do FDID. E uma segunda vertente, que foi trabalhada nos dois últimos anos, é de interação com alunos das escolas estaduais de ensino médio, no sentido de levar o tema para a vivência deles, inserindo um curso de extensão de quarenta horas aos alunos do curso Técnico em Edificações. Os resultados desta segunda abordagem se mostraram muito positivos, tendo repercutido entre os coordenadores pedagógicos das escolas, que solicitaram a continuidade do curso para novas turmas. Com a sistematização dos estudos sobre a acessibilidade, tornou possível o compartilhamento dos conhecimentos com outras áreas de conhecimento com experiências pontuais, para citar, o curso de Pedagogia, na Faculdade de Educação, o de Engenharia Civil e o de Engenharia de Transportes no Centro de Tecnologia. Estas parcerias propiciaram a criação de grupos de estudo e planejamento de ações. A parceria do Departamento de Arquitetura e do Departamento de Fundamentos da Educação aconteceu a partir do Projeto UFC Inclui, com abrangência de várias temáticas e ações, como o laboratório de Acessibilidade para cegos serem capacitados a trabalhar ou 


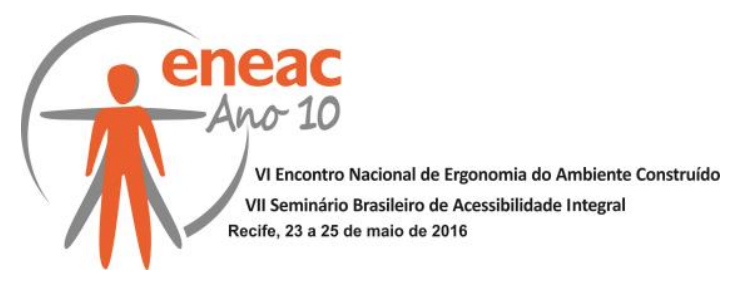

simplesmente aprender a usar o computador; exposições no bosque do $\mathrm{CH} 1$ e adaptações que serão citadas adiante. Importante meio de comunicação e difusão do conhecimento foram os Ciclos de Debates - UFC inclui - Inclusão de pessoas com Deficiência na Universidade Federal do Ceará, organizados desde 2007, a cada ano foi realizado um ciclo de palestras com temas que envolvem a inclusão e acessibilidade, com temas que passam pelos aspectos da infraestrutura física da UFC, dos recursos acessíveis à informática e acesso ao acervo do sistema de bibliotecas, formação de profissionais da saúde para atendimento a pessoas com deficiência, condições pedagógicas para inclusão de alunos com deficiência na UFC: relações interpessoais, metodologia de ensino e avaliação. Em 2009, é criada a Comissão Especial de Educação Inclusiva - CEln, pelo Reitor Jesualdo Pereira Farias, com o objetivo de elaborar proposta de estruturação administrativa que passaria a cuidar em definitivo da elaboração, condução e execução de políticas de Educação Inclusiva nesta Universidade.

Esta comissão elaborou um plano de ações e desenvolveu alguns diagnósticos nos três campi, para subsidiar os argumentos da necessidade de implantar uma política de acessibilidade e inclusão na UFC. Em 2010, foi criada a Secretaria de Acessibilidade - UFC Inclui instituída sob a coordenação da professora Vanda Leitão. Podemos dizer que a culminância das ações pontuais foi a criação desta secretaria como órgão ligado diretamente ao gabinete do Reitor. Os trabalhos da Secretaria se constituem em quatro eixos de atuação - atitudinal, arquitetônico, pedagógico e tecnológico. O eixo atitudinal visa ajudar a comunidade acadêmica a enfrentar o preconceito e incentivar mudanças de atitude; o eixo arquitetônico - visa orientar adequações na estrutura física da UFC; o eixo pedagógico - visa equiparar as oportunidades do aluno com deficiência aos demais colegas, na busca de ações que facilitem o ensino-aprendizagem, com alternativas de avaliação e, o eixo tecnológico - objetiva incentivar pesquisas e ações em tecnologias assistivas, para o desenvolvimento de equipamentos, serviços e estratégias que permitam o acesso ao conhecimento com autonomia. Em 2010, a UFC adquiriu 36 equipamentos - elevadores e plataformas, de modo a beneficiar o acesso as pessoas com deficiência, principalmente de estudantes com deficiência ou mobilidade reduzida. Outras áreas como Engenharia de Telecomunicações, Enfermagem, Odontologia, Fisioterapia também desenvolvem projetos e ações voltadas à acessibilidade e inclusão, e, com o apoio da Secretaria de Acessibilidade foi criado o curso de Licenciatura em Letras-Libras, uma nova vertente no desenvolvimento de estratégias de acessibilidade e inclusão. Outra parceria da Educação e Arquitetura foi o Programa de Educação Inclusiva e Acessibilidade - PEIA, coordenado em 2013 pela Professora Ana Karina Morais de Lira, contou com a participação de alunos de diversos cursos: Arquitetura, Pedagogia, Filosofia, Odontologia e Psicologia. Com a parceria dos Departamentos de Engenharia e de Arquitetura e Urbanismo da UFC foi criado o Grupo de Planejamento e Trabalho em Acessibilidade - GTPA (2007) no Conselho Regional de Arquitetura, Engenharia, Agronomia e Arquitetura - CREA-CE, coordenado pela Professora e Engenheira Nadja Dutra Montenegro. Este Grupo de Trabalho deu seguimento às ações, ampliando para participação de entidades e associações ligadas às pessoas com deficiência, como a Associação dos Cegos do Ceará - ACEC, Instituto Cearense de Educação de Surdos - ICES, Associação dos Deficientes Motores - ADM, dentre outros e mantendo parceria com o Ministério Público Estadual. Foram desenvolvidas várias ações na cidade, inclusive com a parceria da Autarquia municipal de Trânsito - AMC, como passeios acompanhados, multa de alerta nas vagas reservadas na Avenida Beira Mar, simulações de sinalização na Praça do Ferreira no centro da cidade (2007). Além do I Seminário de Acessibilidade (2007) e Seminário Estadual de Calçadas Acessíveis (2010 e 2011).

Outro departamento que compartilha com Arquitetura estudos e pesquisas relacionadas à acessibilidade e inclusão é o de Engenharia de Transportes que oferta, além do mestrado, curso de Especialização em Gestão de Trânsito e Transportes Urbanos, em que algumas monografias apresentadas em 2015 focaram aspectos ligados a acessibilidade sob várias 


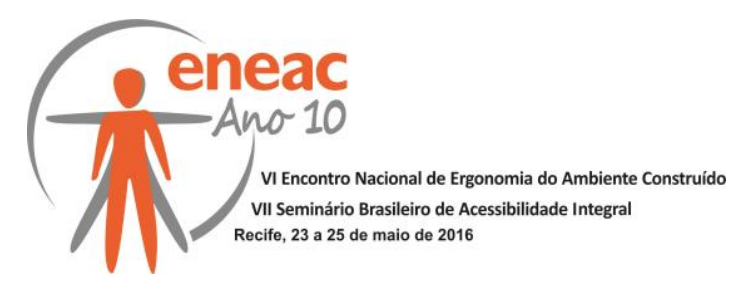

abordagens e foram desenvolvidas sobre minha orientação e da Professora Nadja Dutra Montenegro, sobre os seguintes temas: 1. Condições de vagas destinadas ao estacionamento de idoso: estudo de caso da orla de Fortaleza; 2. Mobilidade e Acessibilidade em Terminal de Ônibus Urbano: estudo de caso - Terminal Antônio Bezerra. 3. Transtornos no Trânsito: o caso do entorno da escola particular Farias Brito no município de Fortaleza; 4. análise da legislação vigente para a garantia da padronização, segurança e acessibilidade na construção e manutenção de calçadas no município de Fortaleza. Quanto às ações institucionais, a Superintendência de Planejamento Físico e Operações PLANOP/UFC iniciou, em 2002, levantamentos das necessidades de adaptação dos campi (Pici, Porangabuçu e Benfica), no que resultou em vários projetos de adaptação que ficaram, de certa forma, a espera de recursos para execução.

Neste sentido, as iniciativas de projetos pontuais, como o Projeto UFC inclui, coordenado pela Professora Ana Karina Morais de Lira, da Faculdade de Educação, tendo obtido financiamento do Proext, veio a colaborar com o planejamento das adaptações da PLANOP, tendo as condições necessárias para executar a maioria dos projetos de adaptação no Centro de Humanidades $-\mathrm{CH} 1$, visto que o projeto destinava-se a este centro, por ter sido apontado em pesquisa de que o maior número de usuários com deficiência na UFC se encontrava neste espaço. Como difusão do conhecimento extra-muros, a UFC é requisitada para apresentar, divulgar e ministrar cursos e palestras nesta área. Assim, foram ministrados cursos em Sobral (2007); Seminário Quixadá Acessível (2008) - Atividades do Grupo de Trabalho em Planejamento e Acessibilidade do Crea-CE. Em Fortaleza foram ministrados cursos no Congresso de Engenharia (2008), no Colégio de Presidentes CREANordeste (2011), para Auditores (Engenheiros e Arquitetos) do Tribunal de Contas do Estado do Ceará - TCE (2012); para os técnicos, arquitetos e engenheiros da Construtora Mota Machado (2013), aos alunos dos cursos de Arquitetura e Urbanismo e de Engenharia Civil membros da Consultec - Empresa Junior do Centro de Tecnologia da UFC (2013), para os técnicos do Tribunal de Justiça em (2014), no Fórum da Cidade da Secretaria Municipal de Urbanismo e Meio Ambiente (2014), na II Semana de Acessibilidade da UFC (2014); para as delegacias regionais do CREA em Quixadá (2008) e Juazeiro do Norte (2009); módulo em curso de Especialização em Gestão de Trânsito/UFC (2013); módulo em curso de Especialização na UNIFOR (2013). Além da elaboração do Guia de Acessibilidade: Espaço Público e Edificações (Montenegro, N.G.S.D.; Santiago, Z. M. P. e Sousa, V.C. de, (2009) e Projeto de Extensão que gerou convênio com o Governo do Estado do Ceará, via Secretaria de Infraestrutura SEINFRA, para diagnóstico das condições de acessibilidade de 92 prédios patrimoniais no Estado do Ceará.

Mais recente, no curso de Arquitetura e Urbanismo, para difusão da temática da acessibilidade espacial, foi introduzida em 2012 a disciplina Desenho Universal, de 64 horas, em caráter optativo. Nela são desenvolvidos estudos de acessibilidade no espaço urbano e nas edificações, o que vem propiciando ampliação dos conhecimentos nesta área de estudo e influenciando as áreas das disciplinas de projeto. Alguns estudos realizados pelo grupo de estudo na área urbana: análise da avenida Bezerra de Menezes, análise da Avenida Monsenhor Tabosa (centro turístico de compras); análise de praças; bem como análise das condições de acessibilidade em edifícios públicos e de uso coletivo, tais como: prédios da UFC, supermercados, centros comerciais, biblioteca pública, clínicas, dentre outros. O desenvolvimento e discussões dos temas na disciplina de Desenho Universal, refletindo sobre o espaço urbano e acessibilidade, nos levou a pesquisar sobre as praças de Fortaleza, analisar as condições de acessibilidade, como projeto PIBIC (2014-2015). Em 2015, com o Programa de Pós-Graduação em Arquitetura, Urbanismo e Design no DAU, foi criada a disciplina Desenho Universal e Acessibilidade no Ambiente construído, abrindo sua primeira turma no primeiro semestre de 2016, implantando a temática como subsídio a pesquisas nesta área. 